\title{
THERMALLY INDUCED MICROMECHANICAL STRESSES IN CERAMIC/CERAMIC COMPOSITES
}

\author{
Zhuang $\mathrm{Li}^{1}$ and Richard C. Bradt ${ }^{2}$ \\ 1 Materials Science Division \\ Argonne National Laboratory, * Argonne, IL 60439 \\ 2 Mackay School of Mines \\ Chemical and Metallurgical Engineering \\ University of Nevada-Reno, Reno, NV 89557-0136 \\ ANL/MSD/CP-78152 \\ DE93 004860
}

November 1992

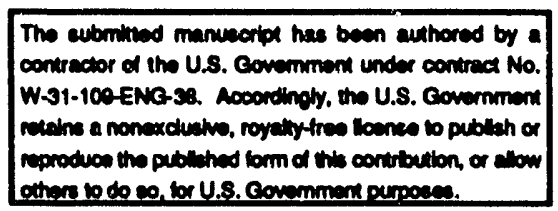

DISCLAIMER

This report was prepared as an account of work sponsored by an agency of the United States Government. Neither the United States Government nor any agency thereof, nor any of their employees, makes any warranty, express or implied, or assumes any legal liability or responsibility for the accuracy, completeness, or usefulness of any information, apparatus, product, or process disclosed, or represents that its use would not infringe privately owned rights. Reference herein to any specific commercial product, process, or service by trade name, trademark, manufacturer, or otherwise does not necessarily constitute or imply its endorsement, recommendation, or favoring by the United States Government or any agency thereof. The views and opinions of authors expressed herein do not necessarily state or reflect those of the United States Government or any agency thereof.

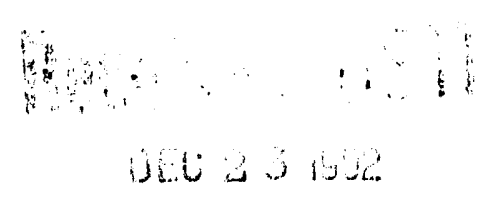

INVITED PAPER presented at the International Confer nce of Thermal Shock and Thermal Fatigue Behavior of Advanced Ceramics, Schloss-Ringberg, Germany, November 9-13, 1992; to be published in the Proceedings, edited by G. Petzow and G. A. Schneider (Kluwer Academic Publishers).

\footnotetext{
"Work supported by the U.S. Department of Energy, BES-Materials Sciences, under contract \#W-31-109-ENG-38.
} 
THERMALLY INDUCED MICROMECHANICAL STRESSES

IN CERAMIC/CERAMIC COMPOSITES

ZHUANG LI ${ }^{(1)}$ AND RICHARD C. BRADT ${ }^{(2)}$

(1) Argonne National Laboratory

Materials Science Division, Bldg. 212

9700 Scuth Cass Avenue

Argonne, IL, 60439, USA

(2) Mackay School of Mines

Chemical and Metallurgical Engineering

University of Nevada-Reno

Reno, NV, 89557-0136, USA

\begin{abstract}
The internal micromechanical stresses which develop in ceramic-ceramic composites as a consequence of temperature changes and thermoelastic property differences between the reinforcing and matrix phases are addressed by the Eshelby method. Results for two whisker reinforced ceramic matrix composites and for quartz particles in porcelain are discussed. It is concluded that the stresses which develop in the second phase reinforcing inclusions are quite substantial (GPa-levels) and may be highly anisotropic in character. These stresses are additive to the macroscopic thermal stresses from temperature gradients which are encountered during heating and cooling, and also to externally applied mechanical stresses (loads). These micromechanical stresses are expected to be highly significant for thermal cycling fatigue and other failure processes.
\end{abstract}

\title{
INTRODUCTION
}

The development of thermal stresses causing thermal stress (shock) damage to ceramic materials, both monolithic ceramics and ceramic/ceramic composites may evolve through the occurrence of several different conditions. In a macroscopic sense, whenever the temperature of a ceramic object or artifact is changed, a thermal gradient develops. As most cerarnics display significant thermal expansion, the ceramic object or artifact experiences a state of thermal stress which relates to the physical properties of the ceramic, including the thermal expansion, Poisson's ratio, elastic modulus, thermal heat transfer characteristics, etc, its geometry and the magnitude of the thermal gradient which develops. This stress development is a form of the ceramic object restraining itself from changing 
dimensions as a result of the thermal gradient. A somewhat extreme of this condition is the specific case where a ceramic object is fixed at its extremities and thus totally restrained from dimensional changes during a uniform temperature change. A simple mechanics analysis of this latter situation reveals that conditions of total restraint can develop very high stresses, often exceeding $100^{\prime}$ 's of MPa for a $\Delta T$ of only a few $100^{\circ} \mathrm{C}$. Of course, the application temperatures of ceramics are much greater than a few $100^{\circ} \mathrm{C}$, thus readily creating severe thermal stress problems in many instances.

On a scale only slightly less than that of the complete ceramic object or artifact itself, yet at a size level which is usually greater than the ceramic microstructure, are the residual stresses which are developed in the glazes on ceramic whitewares during cooling. Although Chinese and Japanese artisans have developed glazes which intentionally yield cracked, or crazed glaze patterns with considerable aesthetic merit, most glazes are usually designed to exist at compressive stress levels of 50-100 $\mathrm{MPa}$ in order to avoid any unsightly cracking. Although the analysis for actually calculating the stresses in glazes may be considerably more complicated than similar calculations for a ceramic monclith, treating the problem as a thin layer on an infinite slab has proven to be satisfactory. Those results indicate that the magnitudes of the thermal stresses which develop in the glaze and the underlying ceramic body relate to the differences in their elastic properties and thermal expansions; as well as to the relaxation temperature of the glaze during cooling and the thickness of the glaze. It is significant that the thickness of the glaze, that is its dimensions, or its geometric relationship to the ceramic body is of importance. This illustrates the significance of the geometric aspects of thermally induced stresses in a manner which is different from the simple thermal gradient effects during heat transfer.

The geometry of the reinforcing phase microstructure is a critical factor in the development of internal thermal stresses in ceramic/ceramic composites. This has been demonstrated for $\mathrm{SiC}$ whiskers reinforcing various matrices ${ }^{(1)}$, and also for $\mathrm{Al}_{2} \mathrm{O}_{3}$ whiskers in both mullite and garnet matrices. ${ }^{(2)}$ The aspect ratio of the whiskers, their length/diameter ratio, has a significant effect. This is particularly true for short whisker, or fiber reinforcements where $(\mathrm{L} / \mathrm{d})$ is less than about five. Equally important are the thermal expansion and elastic anisotropies of the reinforcing phase inclusions for they are invariably single crystals. Since the elastic properties of single crystals are fourth order tensor properties, individual crystal reinforcing phases will be elastically anisotropic. This paper addresses the thermal stresses, or thermally induced micromechanical stresses in ceramic/ceramic composites which are associated with the reinforcing inclusions. It first reviews the Eshelby approach to calculating the thermally induced micromechanical stresses associated with reinforcing phase inclusions, then discusses the results for several ceramic/ceramic 
composites, including $\mathrm{SiC}$ whiskers in alumina, ${ }^{(1)} \mathrm{Al}_{2} \mathrm{O}_{3}$ whiskers in mullite ${ }^{(2)}$ and quartz particles in a porcelain. ${ }^{(3)}$

The conclusions reaffirm that four factors influence the thermally induced micromechanical stresses that are associated with the rain forcing phase within ceramic/ceramic composites. Those factors are:

(i) the thermal expansion mismatch between the phases,

(ii) the elastic modulus mismatch between the phases,

(iii) the geometry of the reinforcing phase or inclusions, and

(iv) the volume fraction of the reinforcing phase.

Of course, if one superimposes externally applied stresses upon a ceramic/ceramic composite, then those applied stresses must add to the internal micromechanical stresses as a second order tensor. Macroscopic thermal stresses from the thermal gradients during rapidly heating or cooling would do likewise. As the internal, thermally induced micromechanical stresses may be quite substantial, in fact they often reach the GPa range, it may be expected that those stresses will make a substantial contribution to mechanical fatigue and also fatigue from thermal cycling. Their variation during thermal cycling can be expected to create local stress levels that far exceed those experienced during normal mechanical cycling from the external design stresses.

\section{MICROMECHANICAL STRESS FORMULATION}

Most research on ceramic reinforced polycrystalline ceramic matrix composites has emphasized the interfacial properties of the reinforcing inclusion phase and the matrix. However, recent studies have demonstrated that the internal micromechanical stresses which are generated by the thermal expansion and elastic moduli differences between the reinforcing inclusion phase and the matrix assume a very important role in the mechanical properties of all ceramic/ceramic composites ${ }^{(4-6)}$. Studies of the microme hanical internal stresses in anisotropic single phase polycrystalline ceramic materials and in multiphase composites have been in progress for several decades. Numerous mathematical models have been proposed. However, among the various models, it is the modifications of the Eshelby equivalent inclusion method ${ }^{(7-10)}$ which appear to be the most promising, even though they are not readily adaptable to describe crystallographically faceted second phase inclusions. ${ }^{(1)}$ One reason for its popularity is that the Eshelby approach can exactly solve the stress field of an anisotropic single crystal grain, or second phase inclusion. It is also relatively straight forward to apply to actual ceramic systems. Using extensions, or modifications of the Eshelby method and its matrix forms, the following related thermoelastic stress problems can be directly addressed and solved: (i) the stresses within an anisotropic single crystal grain, whisker or second phase inclusion, (ii) the stresses at the interface between the second phase inclusion and the matrix, (iii) the average stress within the composite matrix, (iv) the effect, or role 
of the geometry of an anisotropic single crystal inclusion, (v) the effect of the inclusion concentration, or its volume fraction within the matrix and (vi) the change of stresses within the inclusion and matrix by an applied external stress.

Figure 1 schematically illustrates an anisotropic ellipsoidal inclusion that is situated within an isotropic matrix. For the analysis, the thermal expansions and elastic properties of the second phase inclusion are considered to be anisotropic, while those of the surrounding matrix are assumed to be isotropic. The descriptive geometric parameters of the ellipsoidal inclusion are denoted as $L$ and $d$, where $L$ is specified as parallel to the $X_{3}$ axis of the inclusion and the $d$ coincides with the $X_{1}$ and $X_{2}$ axes. The angle $\downarrow$ in Figure 1 is defined as the angle between the $X_{1}$ axis and the direction of interest. Two locations within the matrix, just outside of the inclusion, are of special interest. One is at the equator of the inclusion, $\downarrow=0^{\circ}$, as denoted by point $B$ and the other is at the pole of the inclusion, $\psi=90^{\circ}$, as denoted by point $A$. If the (L/d) ratio of the inclusion is equal to unity, then the ellipsoidal inclusion is spherical. For an (L/d) ratio which is much less than one, the inclusion approximates a flat tabular platelet, and when the $(\mathrm{L} / \mathrm{d})$ ratio is much greater than unity then the inclusion approximates a long cylindrical whisker. Those three special geometric shapes of the inclusion are also illustrated in Figure 1 alongside the inclusion.
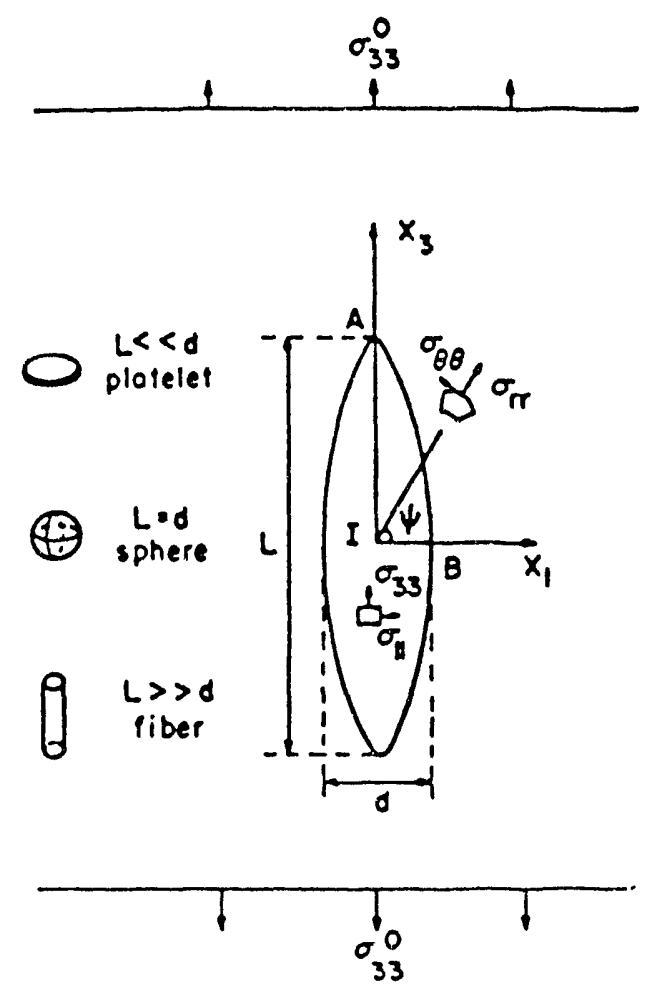

Figure 1. Schematic of an Ellipsoidal Inclusion in a Ceramic Matrix 
Details of the formulation of the micromechanical stress calculations associated with the inclusion in Figure 1 can be found in several references. ${ }^{(7-8)}$ They are presented only in their final matrix form here. To determine the micromechanical internal stresses it is necessary to solve for the eignstrain, $\epsilon_{i j}$, which is generated by the thermal expansion and elastic moduli differences between the second phase inclusion and the matrix. The eigenstrain of the inclusion is expressed in Equation (1) as:

$\left[\left\langle C^{D}\right\rangle\langle S\rangle+\left\langle C^{M}\right\rangle-V_{f}\left\langle C^{D}\right\rangle\langle\bar{S}\rangle\right]\left\langle e^{*}\right\rangle=\left\langle C^{I}\right\rangle\left\langle e^{T}\right\rangle-\left\langle C^{D}\right\rangle\left\langle e^{0}\right\rangle$

where $\left\langle\sigma^{0}\right\rangle,\left\langle\epsilon^{\mathrm{T}}\right\rangle$ and $\left\langle\epsilon^{\circ}\right\rangle$ are each $6 \times 1$ matrices of the applied stress, the thermal strain and the eigenstrain, respectively. The $\left\langle\mathrm{C}^{\mathrm{I}}\right\rangle,\left\langle\mathrm{C}^{\mathrm{N}}\right\rangle$ and $\left\langle C^{D}\right\rangle$ are each $6 \times 6$ matrices of the elastic stiffnesses for the inclusion, the matrix, and the difference between the inclusion and the matrix, respectively. The $\langle\mathrm{S}\rangle$ and $\langle\mathrm{S}\rangle$ are each $6 \times 6$ matrices of the Eshelby tensor and the average Eshelby tensor. These are directly related to the geometry of the inclusion and the elastic properties of the matrix. The $V_{p}$ is the volume fraction of the second phase inclusion. Equation 1 can be considered to be the fundamental equation for the microstructural design of two phase, ceramic reinforced, ceramic matrix composites on a mechanics basis.

The stresses inside of the inclusion are obtained from:

$$
\left\langle\sigma^{\text {m }}\right\rangle=\left\langle\sigma^{0}\right\rangle+\left\langle C^{M}\right\rangle\left[\langle S\rangle-\langle I\rangle-V_{f}\langle\bar{S}\rangle\right]\left\langle e^{*}\right\rangle \text {, }
$$

where $\langle\mathrm{I}>$ is the $6 \times 6$ identity matrix. The average stress in the matrix is determined from:

$$
\left\langle\sigma^{m}\right\rangle=\left\langle\sigma^{o}\right\rangle-V_{f}\left\langle C^{M}\right\rangle\langle\bar{S}\rangle\left\langle e^{*}\right\rangle,
$$

and the stresses just outside of the inclusion in the matrix are expressed by:

$$
\left\langle\sigma^{\text {out }}\right\rangle=\left\langle\sigma^{\text {m }}\right\rangle-\left\langle C^{M}\right\rangle\langle B\rangle\left\langle C^{M}\right\rangle\left\langle e^{*}\right\rangle+\left\langle C^{M}\right\rangle\left\langle e^{*}\right\rangle \text {, }
$$

where $\langle B\rangle$ is related to the elastic constants of the matrix and the unit vector outward from the inclusion.

From the above equations it is evident that the eigenstrain, $\epsilon_{j}{ }^{*}$, is directly related to the thermally induced micromechanical stresses. In Equation (1) it can be seen that the eigenstrain and thus those stresses are influenced by the following four parameters: (i) the difference between the matrix and the inclusion thermal expansions, $\Delta \alpha$, and (ii) their elastic 
moduli difference, $\Delta \mathrm{E}$, (iii) the geometry of the inclusion, expressed as its aspect ratio, (L/d), and (iv) the volume fraction or concentration of the reinforcing phase inclusions, $V_{r}$ The relationships between the eigenstrain and those four composite design parameters are defined through Equation 1. The first and the second terms on the right hand side of Equation $1,\left\langle\epsilon^{\mathrm{T}}\right\rangle$ and $\left\langle\mathrm{C}^{\mathrm{D}}\right\rangle$ respectively, indicate the effects of $\Delta \alpha$ and $\Delta \mathrm{E}$ on the micromechanical stresses. On the left hand side of the Equation 1, the Eshelby tensor $\langle S\rangle$ and $V_{n}$ respectively, indicate the geometrical effects and the effect of the concentration of the second phase inclusions.

Although the formulation of Equations (1-4) appears to be relatively complicated, it has been successfully applied to $\mathrm{SiC}$ whiskers in a polycrystalline alumina matrix and also to sapphire whiskers in garnet and mullite matrices by $\mathrm{Li}$ and Bradt ${ }^{(1,2)}$. For the former, their theoretical calculations are in excellent agreement with the X-ray diffraction experimental residual stress measurements of Abuhasan et al ${ }^{(12)}$ for the same composite in several ways. First, the magnitudes of the internal stresses are nearly the same for the theoretical calculations and the independent X-ray diffraction measur aments. However, more importantly the experimental results substantiate the necessary assumptions for the theoretical calculations, namely that the micromechanical stresses in the inclusions will be highly anisotropic, but those in the polycrystalline matrix are essentially isotropic. Majumdar, et al ${ }^{(6)}$ and Tome et al ${ }^{(13)}$ have employed neutron diffraction techniques to measure the residual stresses within similar $\mathrm{SiC} / \mathrm{Al}_{2} \mathrm{O}_{3}$ composites and their results also confirm the theoretical calculations from applying Equations 1-4. It must be concluded that Equations (1-4) satisfactorily define the thermally induced micromechanical residual stresses in ceramic reinforced ceramic matrix composites.

\section{MICROSTRESSES IN CERAMIC/CERAMIC COMPOSITES}

Application of the Eshelby technique to ceramic composite systems, that is ceramic second phases or inclusions within ceramic matrices, can only be properly applied to those ceramic systems where the single crystal elastic constants have been determined for the reinforcing phase, that is the second phase inclusions. This is, unfortunately, a severe restriction, for the single crystal elastic constants of many crystal structures have never been measured. In addition, because of the anisotropy of the Eshelby formulation, the principal axial thermal expansion coefficients of the inclusion phase are also required. The truth is that only a few systems can be analyzed by this rigorous method, however, those that have been are interesting and have yielded informative results. 
Before addressing those results, it is appropriate, in fact necessary, to briefly address literature calculations which do not fully take into account the complete thermoelastic anisotropy of the inclusions or the second phase. These types of calculations abound in the journals, but none will be singled out for criticism. It must be emphasized that the elastic properties of crystals are fourth order tensors, and thus are anisotropic even for cubic single crystals such as beta-SiC whiskers. In fact, the Young's modulus of cubic $\mathrm{SiC}$ in the $\langle 111\rangle$ is about twice the value in the $\langle 100\rangle$. $^{(14)}$ The failure to incorporate the elastic/thermal anisotropy into calculations of the stresses associated with single crystal inclusions that possess such significant anisotropy can result in very large errors. Analyses which apply only averaged property coefficients for the inclusions and do not fully incorporate the thermoelastic anisotropy must be viewed with considerable reservation.

Figure 2 illustrates the results for the Eshelby analysis applied to three ceramic/ceramic composites, a silicon carbide whisker in a polycrystalline alumina matrix, a sapphire single crystal in a polycrystalline mullite matrix and a quartz particle in a glass matrix. These calculated results are for a single inclusion in an infinite matrix. However, the effects of the volume fraction of the inclusions on the matrix stresses can also be addressed. The reader is referred to the original manuscripts for those effects and others as well. ${ }^{(1-3)}$ Space simply does not permit the presentation of more complete analyses in this manuscript. Nor does it permit further discussions of the thermoelastic anisotropy of the materials. The focus of the remainder of this paper will be on the inclusion associated micromechanical stresses which result from cooling to room temperature after manufacturing at elevated temperatures.

The $\mathrm{X}_{3}$ axes of the reinforcing phase inclusions for these calculations were the [111] for the $\mathrm{SiC}$, the [0001] for the sapphire and also the [0001] for the quartz. The (L/d) ratios as defined in Figure 1 were only examined to a value of five for the quartz, as those particles may be expected to be more of an equiaxed geometry than the single crystal whiskers. The $\Delta \mathrm{T}$ 's were $600^{\circ} \mathrm{C}$ for the quartz porcelain and $1000^{\circ} \mathrm{C}$ for both of the other two composites. The stresses specified in Figure 2 are those as they have been defined in the Figure 1 schematic of the elliposidal inclusion.

There are a number of significant features to these stresses and to the effects of the anisotropies of the second phase inclusions. Several will be addressed in the following discussion. Perhaps the most striking aspect of these stresses is their magnitude, which as it was previously noted, have been confirmed by both X-ray diffraction ${ }^{(5,12)}$ and neutron diffraction. ${ }^{(6,13)}$ The internal stresses in the whiskers are in the GPa range, many times the macroscopic strengths of these composite materials and many times the anticipated design stresses. For the case of $\mathrm{SiC}$ whiskers in alumina, where 

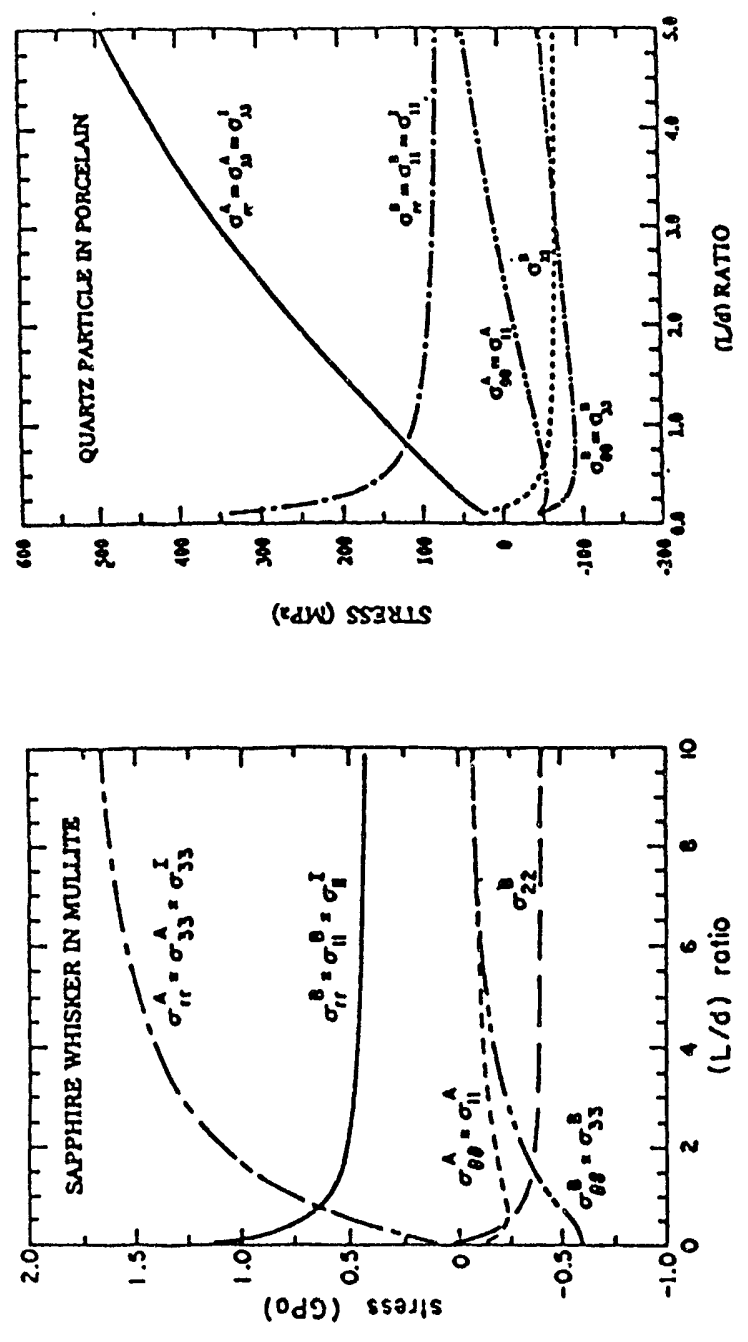

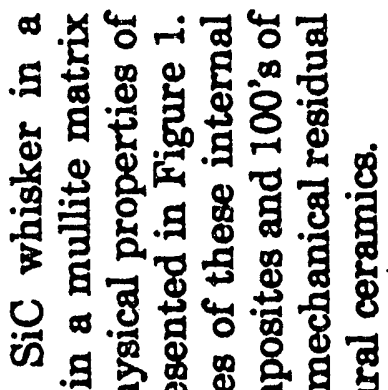

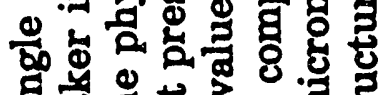

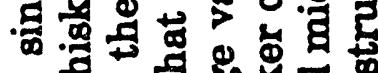
क 5 요.9.

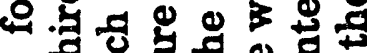

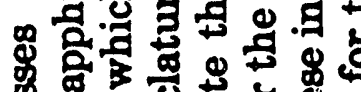

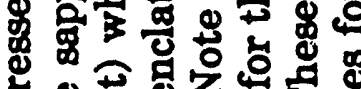

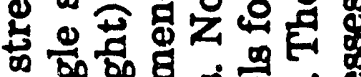
马ี.

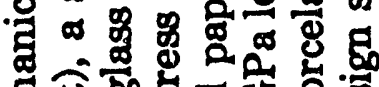

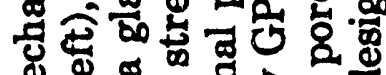

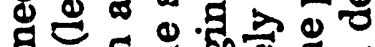

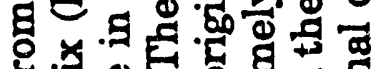
造

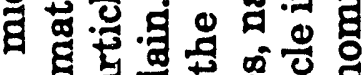

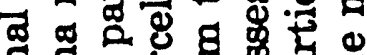

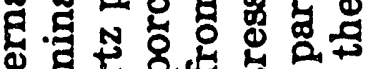

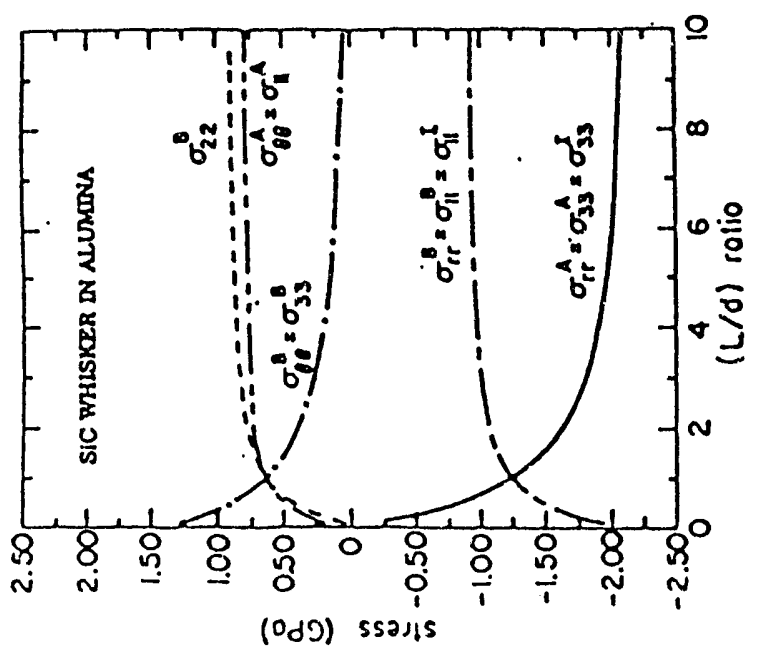

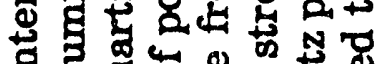
을 年 ง ง

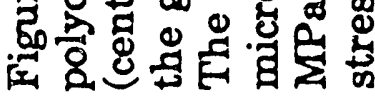


the thermal expansion coefficient of the whisker is less than that of the matrix, the whiskers are in a state of compressive stress of $2 \mathrm{GPa}$ along their longitudinal axis and $1 \mathrm{GPa}$ in the radial direction. It has been suggested that these very high compressive residual stresses in the whiskers make a significant contribution to the room temperature toughness of these $\mathrm{SiC}$ whisker reinforced polycrystalline alumina ceramic matrix composites. One can hardly dispute that hypothesis for that composite system is the only one which has experienced widespread commercial success to date. ${ }^{(15)}$

Alumina or sapphire whiskers reinforcing mullite is an extremely attractive composite system from the thermodynamic stability point of view, as $3 \mathrm{Al}_{2} \mathrm{O}_{3} \cdot 2 \mathrm{SiO}_{2}$ and $\mathrm{Al}_{2} \mathrm{O}_{3}$ are in equilibrium in air to temperatures above $1700 \cdot \mathrm{C}$. It is unfortunate that there is such an unfavorable thermal expansion mismatch in this system, one which creates significant tension within the reinforcing sapphire whiskers. Sapphire whiskers in a mullite matrix would develop longitudinal tensile stresses of over one $\mathrm{GPa}$, a value which is well in excess of their reported tensile strengths, thus fracturing the whiskers as reported by Mah et al. ${ }^{(16)}$ It is a prediction which can be made from the previously outlined Eshelby stress calculations without ever actually manufacturing the composite.

Both of these whisker composite systems exhibit a strong $(\mathrm{L} / \mathrm{d})$ dependence of the internal micromechanical stresses. These internal stresses reach an asymptotic value between (L/d) ratios of about six to ten. This result sends a strong message to the microstructural designers of these types of composites, for it reveals that there is little, or nothing, to be gained by putting very long fibers in these structures, when and if the mechanical property enhancement is based on the residual micromechanical stress state of the fibers. Furthermore, since the processing of powderwhisker (fiber) systems is quite difficult for long fibers, there is really no need to go to extremes to process very long fiber composite systems. An $(\mathrm{L} / \mathrm{d})$ ratio of six to ten is adequate.

The effect of the (L/d) ratio on the residual micromechanical stresses in these two fiber systems illustrates a significant feature for all whisker composites. This geometric effect is included within the matrix of the Eshelby tensor, $\langle\mathrm{S}\rangle$, in Equation 1. One type of whisker (SiC) is highly elastically anisotropic, but it is isotropic in its thermal expansion, whereas the other whisker (sapphire) is much less anisotropic elastically, but possesses a much greater thermal expansion anisotropy. In spite of this contrast, both exhibit very similar stress versus (L/d) profiles, albeit one is in compression and the other is in tension. This very vividly illustrates that the geometry of the reinforcing inclusion phase has a profound effect on the residual stress state. That effect is superimposed on the thermoelastic anisotropy of the crystal structure of the inclusion. The form of the rapid 
increase of the micromechanical stresses for platelet geometry $(\mathrm{L} / \mathrm{d})$ ratios of less than one suggests extreme caution to those designers which attempt to use platelets for composite reinforcements, particularly when the platelets have a greater thermal expansion than the matrix.

The stresses within and about a quartz particle in a typical tri-axial porcelain are also presented in Figure $2 .^{(3)}$ These are quite interesting in several respects. It is well known that traditional quartz-porcelains are prone to internal microcracking about the quartz particles. ${ }^{(17)}$ These are partially spherical cracks which appear to parallel the particle quartz surface just removed from the particle into the glass matrix. These cracks are caused by the $\sigma_{\mathrm{rr}}$ stress in Figure 1, which Figure 2 reveals is a tensile stress that can reach a level of several hundred $\mathrm{MPa}$. The Eshelby calculations suggest that these cracks will most likely occur perpendicular to $<2110\rangle$ directions for platelet shaped quartz grains, but perpendicular to the $<0001\rangle$ for more fiber shaped or elongated quartz grains. All of the micromechanical stresses for the quartz/porcelain system appear to reach a symptotic values at low (L/d) particle ratios, except for the tensile stress in the $<0001>$ which increases nearly linearly beyond an $(\mathrm{L} / \mathrm{d})$ value of five. This further illustrates the geometrical/anisotropic coupling of the micromechanical stresses associated with second phase inclusions.

An important feature of these very high micromechanical stresses is their direct association with the reinforcing phases and the inclusion/matrix interface. The character of the interface between the matrix and the reinforcing whiskers or particles is well known to be a critical design parameter for virtually all composites. Its proximity to these very high stresses and the high strain energy density of the inclusion and the immediate surrounding matrix is particularly critical, potentially increasing its susceptibility to localized degradation phenomena. If, for example, one is interested in the effects of externally applied stresses on the cyclic fatigue of these composites, and the mechanism is one of interfacial debonding, failure or cracking adjacent to a reinforcing inclusion, then it is necessary to consider the internal micromechanical stresses as well as the externally applied macroscopic stresses. This synergism can be addressed by the Eshelby method as it has been formally outlined by Li and Bradt ${ }^{(1)}$ in their original paper on $\mathrm{SiC}$ whiskers in polycrystalline alumina.

\section{SUMMARY AND CONCLUSIONS}

Application of the Eshelby method to the calculation of the internal micromechanical stresses which develop in ceramic/ceramic composites during temperature changes is outlined. The methodology is then applied to two ceramic whisker reinforced ceramic matrix composites and to quartz in porcelain for the determination of the residual stresses which result from cooling to room temperature after elevated temperature processing. Several 
practical consequences of these types of theoretical calculations are then discussed.

The general Eshelby derived equation clearly establishes the physical and structural characteristics of ceramic matrix composites with ceramic reinforcing second phase inclusions that affect the internal micromechanical stresses. There are: (i) the thermal expansion mismatch, (ii) the elastic modulus mismatch, (iii) the geometry of the reinforcing inclusion phase, and (iv) the volume fraction of the reinforcing phase. The residual stresses which result from cooling to room temperature after processing at elevated temperatures can be in the GPa range, a level which is far beyond the macroscopic design stresses of structural ceramics. As these very high stress levels are directly associated with the reinforcing phases and the inclusion/matrix interface, they may be instrumental in promoting failure.

A significant geometric effect occurs as the micromechanical stresses associated with whisker reinforcement 3 exhibit a strong (L/d) dependence. The stresses reach asymptotic level between $(\mathrm{L} / \mathrm{d})$ values of six to ten in most cases. This result suggests that it may not be necessary to utilize long whiskers or fibers in these types of composites, a welcome conclusion since long whiskers or fibers are difficult to process into fully dense composites. The rapid changes of the micromechanical stresses near the platelet geometry for an inclusion also suggests that the optimal design of particulate reinforced ceramic/ceramic composites may be considerably more difficult to achieve than for whisker reinforced composites.

\section{ACKNOWLEDGEMENTS}

The analysis presented in this paper was developed under the support of NASA through Grant NAGW - 199 and by the USDOE under Basic Energy Sciences - Materials Sciences Contract W-31-109-ENG-38. The authors acknowledge technical discussions of the principles presented here with P. Predecki and M. Taya.

\section{REFERENCES}

1. Li, Z. and Bradt, R.C., (1989), "Micromechanical Stresses in SiCReinforced Alumina Composites", J. Amer. Cer. Soc. 2, (1), 70-77.

2. Li, Z. and Bradt, R.C., (1992), "Micromechanical Stresses in Sapphire Whisker and Alumina Fiber Reinforced Mullite and Garnet Ceramic Matrix Composites", J. Eur. Cer. Soc. $\underline{9}$, (3), 143-152.

3. Li, Z. and Bradt, R.C. "Residual Stresses in Quartz and Alumina Porcelains" (to be published). 
4. Li, Z and Bradt, R.C., (1988), "Thermoelastic Anisotropy in SiC, Residual Stresses in Monolithic SiC Ceramics and SiC Whisker Reinforced Composites, "pp. 511-523, in Materials Science Forum, Vol. 34-36 Edited by C.C. Sorrell and B. Ben-Nissan, Trans Tech Pub., Switzerland.

5. Predecki, P. K, Abuhasan, A. and Barrett, C.S., (1988), "Residual Stress Determination in $\mathrm{Al}_{2} \mathrm{O}_{3} / \mathrm{SiC}$ (Whisker) Composites by X-Ray Diffraction," pp. 231-243 in Advances in X-Ray Analysis, Vol. 31 Edited by C.S. Barrett, J.V. Gilfrich, R. Jenkins, J.C. Russ, J.W. Richardson, Jr and P.K. Predecki, Plenum Press, New York.

6. Majumdar, S., Kupperman, D. and Singh, J.P., (1988), "Analytical and Experiunental Determination of Residual Thermal Stresses in a Ceramic-Ceramic Composite," J. Am. Ceram. Soc., 71 [10] 858-863.

7. Eshelby, J.D., (1957), "The Determination of the Elastic Field of an Ellipsoidal Inclusion and Related Problems", Proc. Roy. Soc. (London) A241, 376-396.

8. Mura, T. (1982), pp. 66-75, in Micromechanics of Defects in Solids, Martinus Nijhoff Publishers, The Hague.

9. Khachaturyan, A.G., (1983), pp. 226-240 in Theory of Structural Transformations in Solids, John Wiley \& Sons, New York, N.Y.

10. Taya, M. (1981), "On Stiffness and Strength of an Aligned Short Fiber Reinforced Composite Containing a Penny-Shaped Crack in the Matrix," J. Comp. Mater., 15 198-210.

11. Rao, S.S., Tsakalakos, T., and Cannon, R.W. (1992), "Stress Distribution in Ceramic Composites Containing Faceted Inclusions", J. Amer. Cer. Soc. 75, (7), 1807-1817.

12. Abuhasan, A., Balasingh, C. and Predecki, P. K. (1990), "Residual Stresses in Alumina/Silicon Carbide (Whisker) Composites by X-ray Diffraction", J. Amer. Cer. Soc. $7 \underline{3}$ (9) 2474-2484.

13. Tome, C.N., Bertinetti, M.A. and MacEwen, S.R (1990), "Correlation Between Neutron Diffraction Measurements and Thermal Stresses in a Silicon Carbide/Alumina Composite", J. Amer. Cer. Soc. $\underline{73}$ (9), 3428-3432.

14. Li, Z. and Bradt, R.C., (1987), "The Single Crystal Elastic Constants of Cubic (3C) $\mathrm{SiC}$ to $1000 \cdot \mathrm{C}^{n}$, J. Mat. Sc. $\underline{22}, 2557-2559$. 
15. Homeny, J., Vaughn, W.L., and Ferber, M.K. (1987), "Processing and Mechanical Properties of SiC-Whisker $\mathrm{Al}_{2} \mathrm{O}_{3}$-Matrix Composites", J. Amer. Cer. Soc. 70, (6), 393-395.

16. Mah, T., Mendiratta, M.G. and Boothe, L., (1988), "High Temperature Stability of Refractory Oxide-Oxide Composites", Tech. Rpt. AFWALTR-88-4015.

17. Warshaw, S.I. and Seider, R, (1967), "Comparison of Strengths of Triaxial Porcelains Containing Alumina and Silica", J. Amer. Cer. Soc. 50, (7), 337-342. 

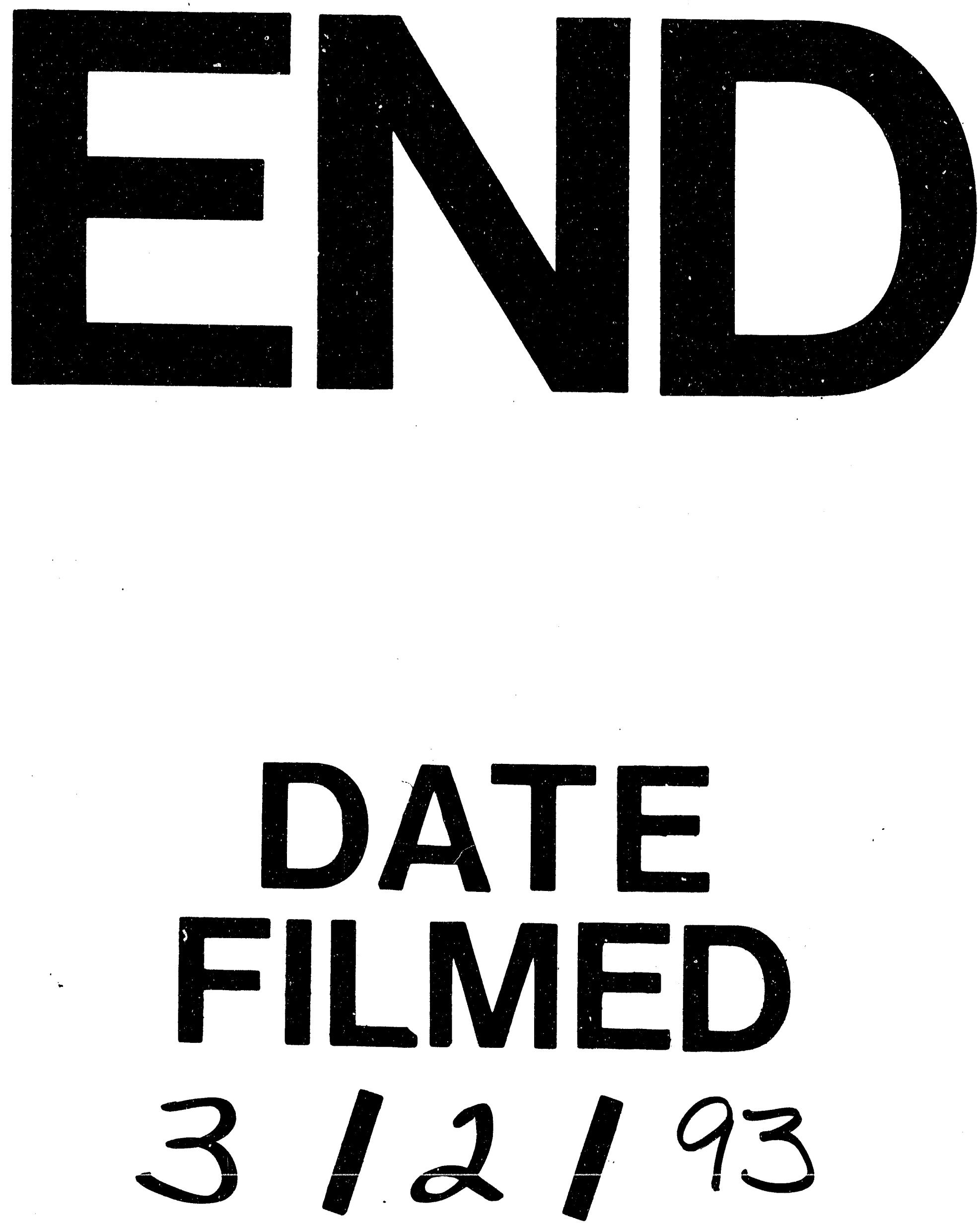
